\title{
TOTALLY TUBELESS PERCUTANEOUS NEPHROLITHOTOMY (PCNL) : IS IT FEASIBLE IN MORBIDLY OBESE PATIENTS ?
}

\section{Rahul Goel*}

\author{
Associate professor, Surgery Department of Surgery, Muzaffarnagar \\ Medical College, Muzaffarnagar. ${ }^{*}$ Corresponding Author
}

\section{ABSTRACT PURPOSE - PCNL is difficult in obese, and has increased risk of peri-operative complications in obese, it} was decided to retrospectively analyse from our patients, of last $8 \mathrm{yrs}$, to determine stone free status and complication rates.MATERIAL AND METHODS - Study was between January 201 1- January 2019 , 78 morbidly obese patients were randomised, with body mass index over 35 who had indication of PCNL, stones larger than $2 \mathrm{cms}$ and stones smaller in size and treatment unfit for ESWL (extra corporeal shock wave lithotripsy) if stone skin difference was high, or ESWL resistant hard stones were enrolled. Patients randomly assigned in two groups, Group 1 standard PCNL with Nephrostomy and a Stent ,Group 2 Totally tubeless with no Nephrostomy and no Stent, and the outcomes were compared. RESULTS - The transfusion rate, operative time and the drop in hemoglobin were same in both groups ( $p>0.05$ ). Total analgesic use was equivalent of 33.8 vs $14.7 \mathrm{mgs}$ of morphine sulfate $(18-77 \mathrm{mg})$ and was significantly lower in tubeless group $(\mathrm{p}=0.001)$. Return to normal was described as total number of in-patients and outpatients days from time of admission to the point which the patient returns to normal activity such as going to job and was 19.4 vs 9.3 days (6-30 days, $\mathrm{p}=0.001)$ CONCLUSION - Totally tubeless PCNL in obese patients had lower analgesic use and return to normal activity vs standard PCNL. Totally tube less PCNL is recommended for obese patients.

\section{KEYWORDS :Obesity ,Percutaneous Nephrolithotomy}

\section{INTRODUCTION}

Obesity is one of the risk factors for renal stone disease, and bears higher surgical risk to patients and surgeons, leading to more complications both surgical and anaesthetic (2-3) .ESWL is not recommended in obese due to increased skin stone distance leading to poor stone fragmentation(4). Totally tubeless PCNL (no nephrostomy no stent) was shown to be a better alternative in selected patients, but it has been used quite often now, and more indications for its use continue to emerge (5-6).

Regarding technical difficulties that obesity might impose on PCNL success and the high risk of perioperative complications in this group of patients, we retrospectively gathered data of our patients during last 8 years to determine the stone free and complication rates.

\section{MATERIAL AND METHODS}

Between January 2011 to January 2019, 78 obese patients with body mass index (BMI) over 35 , having indication of PCNL ( stone more than $2 \mathrm{cms}$, smaller stones resistant to ESWL, or patients not fit for ESWL due to increased skin stone distance) , and with no contraindication for PCNL ( bleeding diathesis , inability to position in prone position) were counselled for PCNL and an informed consent was obtained.

Patients were randomly assigned to groupl (standard PCNL with both nephrostomy and stent) and group 2 (totally tubeless PCNL with no nephrostomy and stent). PCNL was done in prone position under image intensifier guidance, standard 26 fr tract was made and standard 24fr Storz Nephroscope was used. Due to thick fat the Amplatz sheath was tagged to skin with suture to facilitate withdrawal of Amplatz in case of inward migration of Amplatz in thick fat. At the end the patients were randomised in group 1 and group 2 .

Age ,stone burden ( $\mathrm{cm}^{2}$ according to computed tomography scan), hemoglobin drop, total morphine and analgesic use, $\mathrm{BMI}$, time to return to normal activity stone free rates were recorded.

Patients with perforation of pelvi-calyceal system and need for more than one tract were excluded from study.

\section{STATISTICAL ANALYSIS}

Oneway ANOVA and PAWS v 18 was used for data gathering and analysis

\section{RESULTS}

78 patients were enrolled , 43 in group 1 (standard PCNL -stent and nephrostomy used) and 35 in group 2 (totally tube less PCNL , no stent and nephrostomy), 23 females and 55 males, mean age was 44.3 and 42.3 yrs $(p=0.57)$, mean stone burden was respectively 9.4 and $9.3 \mathrm{~cm}^{2}$ ( range $2-31, \mathrm{p}=0.9$ ) and BMI was 35.3 vs.35.7 ( $p=0.3195 \%$ CI $34.8-35.8$ ). Only 6 patients in group 1 , and 2 patients in group 2 needed blood transfusion which was not significant between groups $(p=0.21)$. Average hemoglobin drop was 2.1 and $1.7 \mathrm{mg} / \mathrm{dl}$ (maximum $4.5 \mathrm{mgs} / \mathrm{dl}$ ) $\mathrm{p}=0.08(95 \%$ CI 1.7-2.0), stone free rate was $89.3 \mathrm{vs} 89.2$ ( $\mathrm{p}=0.9$, 95\%CI 86.5-92.1)

Operation time was not different between two groups and was between (35.0 and 165.0) minutes in group $l$ and (40 to 185.0 ) minutes in group $2(\mathrm{p}=0.7)$

Total analgesic use was equivalent of $33.8 \mathrm{vs} 14.7 \mathrm{mgs}$ of morphine sulfate (18-77mgs) and was significantly lower in total tubeless group $(p=0.001$.)

Return to normal activity was described as total number of inpatient and outpatient days from time of admission to the point which the patient returns to normal life activity such as going to work was 19.4 vs 9.3 days ( $6-30$ days, $p=0.001$ )

\section{DISCUSSION}

PCNL in obese patients is associated with higher intra operative complications, the stone free rates and complications rates among obese patients under totally tubeless and standard PCNL were compared, it was seen that return to normal activity and analgesic use were significantly lower in patients under totally tube less procedure. However the transfusion rate, operating time and the drop in hemoglobin were same in both groups.

In the study by Yang et al (7) 133 patients under tubeless were enrolled, including $24.9 \%$ obese patients and the success rate was same in obese and nonobese patients, in fact the obese patients demonstrated good out come in this study. Agrawal et al (8) reported in a review study that PCNL results are not related to $B M I$ and obesity among under-operation 
subjects.(9)

Kuntz et al(9) assessed 268 subjects under tubeless PCNL and reported no association between outcomes and reported no effect by obesity on therapeutic outcomes as shown in our study. Another review study by Agarwal et al (10) demonstrated the same results in obese and non obese patients in tubeless procedure.

Alyami et al(11) reported in 114 patients under PCNL that BMI did not contribute in outcomes. However no clinical trial was done in obese subjects under totally tubeless PCNL.

As per our study totally tube less PCNL in obese patients required lower analgesic use and return to normal activity earlier in comparison to standard PCNL, we thus recommend totally tubeless PCNL in obese patients. Though more studies with large sample size and multi-centre sampling is required to attain more definite results.

\section{REFERENCES}

1. Taylor EN, Stampfer MJ , Curham GC. Dietary factors and the risk of incident kidney stones in men: new insights after 14 yrs of follow up J Am Soc Nephrol 2004:15:3225-32

2. Alwan A, Global status report on noncommunicable diseases 2010.2011:world Health Organisation.

3. Bagrodia $A$. Impact of body mass index on cost and clinical outcomes after percutaneous nephrolithotomy. Urology 2008:72;756-60

4. Hammad FT, Balakrishnan A. The effect of fat and non fat components of the skin to stone distance on shockwave lithotripsy outcome.J Endourol 2010:24:1825-9

5. Aghamir SM, Modaressi SS, Aloosh M, Tajik A. Totally tubeless percutaneous nephrolithotomy for upper pole renal stones using subcostal access. I Endourol 2011:25:583-6.

6. Aghamir SM, Mohammadi A, Mosavibahar S, Meysamie A. Totally tubeless percutaneous nephrolithotomy in renal anomalies .J Endourol 2008;22:2131-4

7. Yang RM ,Bellman GC ,Tubeless percutaneous renal surgery in obese patients .Urology 2004:63:1036-41

8. Agrawal MS, Agrawal M. Percutaneous nephrolithotomy :large tube ,small tube, tubeless or totally tube less? Indian J Urol2013:29:219-24

9. Kuntz NJ, Neisius A, Astroza GM. Does body mass index impact the outcomes of tubeless percutaneous nephrolithotomy? BJU Int 2014:114:404-11

10 Agrawal MS ,Agrawal M. Tubeless percutaneous nephrolithotomy .Indian I Urol 2010:26:16-24

11. Alyami FA, Skinner TA, Norman RW. Impact of body mass index on clinical outcomes with percutaneous nephrolithotomy .Can Urol Assoc J 2013;7:197201 